\title{
SYNTHESIS AND CHARACTERIZATION OF TOLUENE SULFONIC ACID (TSA)- DOPED POLYPYRROLE NANOPARTICLES: EFFECTS OF DOPANT CONCENTRATIONS
}

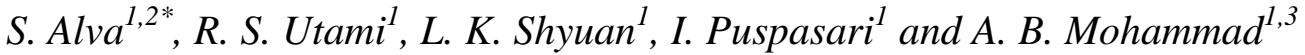 \\ ${ }^{1}$ Fuel Cell Institute, Universiti Kebangsaan Malaysia, 43600 UKM Bangi, Selangor, MALAYSIA \\ ${ }^{2}$ Mechanical Engineering Department, Faculty of Engineering, Mercu Buana University, Jl. Meruya Selatan, No 01, West \\ Jakarta, 11650, Jakarta,-INDONESIA \\ ${ }^{3}$ Department of Chemical Engineering and Process Engineering, Faculty of Engineering and Built Environment, \\ Universiti Kebangsaan Malaysia, 43600 UKM Bangi, Selangor, MALAYSIA
}

\begin{abstract}
Nanoparticles of the conducting polymer polypyrrole in toluene sulfonic acid (PPy/TSA) were synthesized and characterized. The polymerization was process carried out in situ using ammonium persulfate (APS) as an oxidant. The particles were synthesized by varying the dopant concentration of para-toluene sulfonic acid over five sulphonic acid concentrations. The main objective of this study was to examine the effect of TSA dopant concentrations on the properties of polypyrrole nanoparticles. Understanding nature and characteristics of polypyrrole/TSA nanoparticles are important in determining whether the nanoparticles have the potential to be a component in the manufacture of fuel cells. The conducting polymer particles synthesized in this study were characterized using a particle analyzer, X-ray diffraction (XRD), scanning electron microscopy (SEM), Fourier transform infrared spectroscopy (FTIR), spectroscopy UV-visible (UV-vis), thermogravimetric analysis (TGA) and electrical conductivity measurement. XRD shows that the particles generated possessed an amorphous structure, as also indicated by SEM images revealing the formation of aggregated and granular composite particles. Furthermore, the FTIR peak between 1273 and $1283 \mathrm{~cm}^{-1}$ indicated that sulfonic acids $\left(\mathrm{SO}_{3}{ }^{-}\right)$groups were present in the structure of PPy. The size of the PPy/TSA nanoparticles was determined to be approximately $24-51 \mathrm{~nm}$, and their conductivity measured to be $1.3 \times 10^{-1} \mathrm{~S} / \mathrm{cm}$.
\end{abstract}

Keywords: nanoparticle, polypyrrole, conducting polymer, sulfonic acid, conductivity

*Corresponding author: Tel. +62 215840816 ext. 2751/5200

E-mail address: sagir.alva@mercubuana.ac.id

\section{Introduction}

Conducting polymers have been widely used in diverse applications owing to their attractive physical and chemical properties. Among such polymers, polypyrrole (PPy) has garnered extensive research and commercial attention due to its relatively high conductivity, novel electrochemical and optical properties, good biocompatibility, and ease of synthesis [1]. Applications of PPy can be found in semiconductor and electronic devices such as biosensors, gas sensors, wires, microactuators, solid electrolytic capacitor, electrochromic windows and displays, polymeric batteries, and functional membranes [2].In addition, PPy and its composites have also found application in fuel cells, for example, as catalyst supports for DMFCs [3] and PEMFCs [4], anodes for microbial fuel cells [5], and membranes for DMFCs [6].

PPy is generally synthesized by electrochemical or chemical polymerization. Several researchers have investigated the effects of various factors on the properties of polypyrrole synthesized using these methods. Wang et al. [7] synthesized PPy nanomaterials via chemical oxidative polymerization using acid blue AS (AS) as the dopant and surfactant. The authors observed that upon increasing the feeding ratio of AS, the morphology of the resultant PPy/AS nanomaterial changed from nanoparticles to clusters of nanofibers and nanoparticles and, then to nanorods, by contrast, micron-sized PPy particles were formed in the absence of AS. Upadhyay and Kumar [8] reported on the synthesis of PPy nanotubes of varying diameters using the reactive self-degraded $\mathrm{MO}$ $\mathrm{FeCl}_{3}$ template method in the presence of the cationic surfactant CTAB. Their results showed that the diameter of the nanotubes decreased with increasing CTAB concentration. Hazarika and Kumar [9] synthesized PPy nanoparticles by chemical oxidative micro-emulsion polymerization 
in the presence of ammonium persulfate (APS) as an oxidant with varying concentrations of sodium dodecyl sulfate (SDS) surfactant. Their results showed that the shape of the PPy nanoparticles changed from spherical to an elongated shape when the SDS concentration was increased above its second critical micelle concentration $(0.2 \mathrm{M})$. Other characteristics observed in response to the increase in the SDS concentration were a decrease in the optical band gap energy, and an increase in the thermal stability of the PPy nanoparticles.

It has been demonstrated that the application of a macromolecular proton acid as a dopant in the synthesis of conducting polymers, can enhance the electronic conductivity of the resulting material [3, 10].Currently, the effect of the doping concentration of TSA on the nature and characteristics of polypyrrole nanoparticles has not been extensively studied. However, such research is important, to determine whether TSA-doped nanoparticles with have the potential of serving as a component of fuel cells. In the present study, we and investigated the effect of TSA as a dopant on the properties of synthesized PPy nanoparticles.

\section{Experimental}

\subsection{Materials}

Pyrrole purchased from EMD Millipore Co. was used without distillation. Ammonium persulfate (APS) was purchased from Calbiochem and used as an oxidant. Toluene-4- sulfonic acid (TSA) monohydrate was purchased from EMD Millipore Co. and used as a dopant. Analytical grade ethanol was used without purification. Deionized water (DIW) was used in all procedures.

\subsection{Methods}

\subsubsection{Preparation of PPy/TSA}

Polypyrrole (PPy) was prepared by a simple chemical oxidative polymerization method using APS as an oxidant; chemical polymerization was carried out by combining a $0.1 \mathrm{M}$ aqueous solution of pyrrole and a $0.4 \mathrm{M}$ solution of APS. First, TSA (concentration: (a) $0.0 \mathrm{M}$, (b) $0.01 \mathrm{M}$, (c) $0.05 \mathrm{M}$, (d) $0.1 \mathrm{M}$, and (e) $0.5 \mathrm{M}$ ) was added to $100 \mathrm{~mL}$ of DIW in a $250 \mathrm{~mL}$ round-bottom flask and stirred for 15 min. Fresh pyrrole was added to abovementioned solution and stirred for $45 \mathrm{~min}$ under ice-cold conditions to obtain a white turbid mixture. The polymerizing conditions are summarized in Table 1 . APS $(0.9 \mathrm{~g})$ dispersed in $10 \mathrm{~mL}$ DIW was then added dropwise to the white turbid mixture, and stirring was continued for $7 \mathrm{~h}$ under ice-cold conditions. The product of the process was a black precipitate, which was further washed and filtered several times using DIW and ethanol until the filtrate was colorless to remove any possible oligomers and then dried under vacuum for $24 \mathrm{~h}$ at room temperature. The obtained black powder was further ground in an agate mortar and pestle to yield a fine PPy powder.

\subsubsection{Analysis and Measurements}

FTIR analysis was carried out using Fourier transform infrared-near infrared (FTIR-NIR) spectroscopy on a Perkin Elmer spectrophotometer at room temperature over the frequency range of $4000-650 \mathrm{~cm}-1$. The samples were analyzed using the ATR method. Wide-angle X-ray diffraction (XRD) measurements were carried out on a Bruker/D8 Discover X-ray diffractometer over a scattering angle $(2 \theta)$ range of $10-60^{\circ}$ using $\mathrm{CuK} \alpha$ radiation with a wavelength $\lambda=1.504 \AA$. UV-vis absorption spectra were obtained on a Perkin Elmer/Lamda $35 \mathrm{UV}$-vis spectrometer using a $1 \mathrm{~cm}$ quartz cuvette.

The morphology of the PPy nanoparticles was examined using a variable pressure scanning electron microscope (VPSEM) with a ZEISS EVO MA 10 (UK) energy dispersive X-ray (EDX) and an EDAX APOLLO X (USA) model. The particle size of the TSA-doped polypyrrole samples at different molar ratios was measured using a Malvern Zetasizer model Nano zs/ZEN 3600. Thermogravimetric analysis (TGA) of TSA-doped PPy was recorded in the temperature range $30-800^{\circ} \mathrm{C}$ at a heating rate $10^{\circ} \mathrm{C} / \mathrm{min}$ a using Netzsch/STA 449 F3 Jupiter analyzer.

The proton conductivity of the synthesized PPy powder was measured by electrochemical impedance spectroscopy (EIS) over a frequency range of $10^{2}$ to $10^{4}$ at $10 \mathrm{mV}$ and room temperature. The proton conductivity was measured with a two-point probe cell. The above mentioned powder samples were crushed and finely ground in an agate mortar. The powders were pressed into pellets with a diameter of $13 \mathrm{~mm}$ and different thicknesses by applying pressure of $3000 \mathrm{~Pa}$ in a hydraulic press. The proton conductivity could be calculated by the relation $\sigma=$ L/RA, where L and A are the thickness of the sample and the surface area of the electrode, respectively, and $\mathrm{R}$ is the resistance determined from impedance data.

\section{Results and Discussion}

\subsection{FTIR Analysis}

Fig. 1 shows the FTIR spectra of the PPy/TSA samples over the frequency range of $4000-650 \mathrm{~cm}^{-1}$. 
The positions of the absorption peaks were confirmed by comparison with previous reports concerning PPy [11-13].
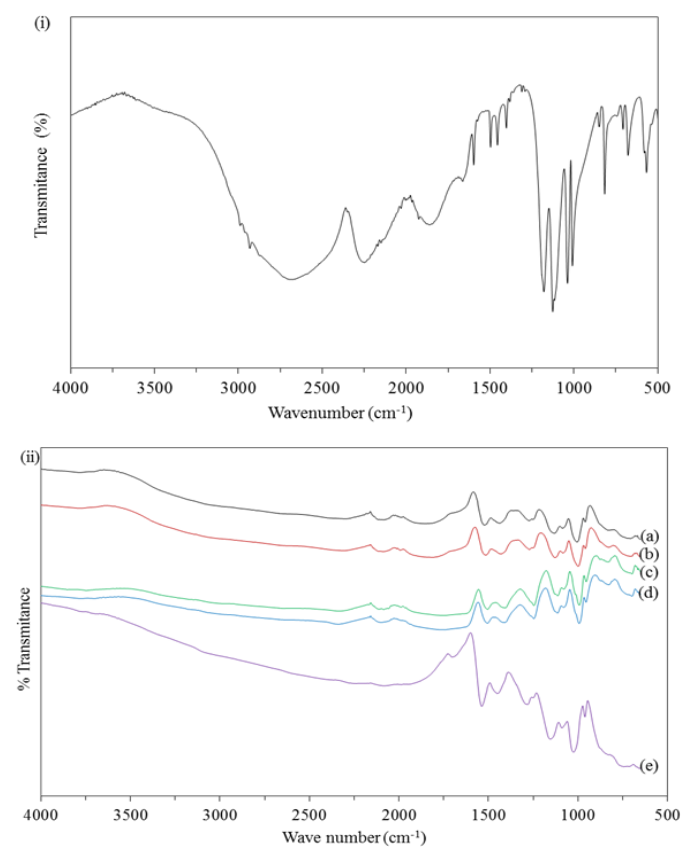

Fig. 1. FTIR Spectra of (i) TSA and (ii) PPy undoped (a); PPy synthesized at $0.01 \mathrm{M}(\mathrm{b}), 0.05 \mathrm{M}(\mathrm{c}), 0.1 \mathrm{M}(\mathrm{d})$, and $0.5 \mathrm{M}(\mathrm{e}) \mathrm{TSA}$ concentrations

The spectrum of TSA is also presented for comparison. The FTIR spectra of doped PPy and TSA showed significant differences. TSA exhibited characteristic bands at 2684, 2248, and $1858 \mathrm{~cm}^{-1}$ (Fig 1. (i)), whereas PPy demonstrated stretching bands at $1537 \mathrm{~cm}^{-1}$ due to the pyrrole ring. Peaks within the range $1811-1336 \mathrm{~cm}^{-1}$ are assigned to aliphatic $\mathrm{C}-\mathrm{H}$ stretching mode vibrations, depending on the TSA concentration, and the stretching band at $1451 \mathrm{~cm}^{-1}$ is attributed to aromatic $\mathrm{C}=\mathrm{C}$ vibrations in polypyrrole. The strong absorbance peaks observed within the range $1273-1283 \mathrm{~cm}^{-1}$ in the PPy/TSA spectra indicate the coupling of $\mathrm{S}=\mathrm{O}$ with the stretching vibration of the pyrrole ring [7, 14].

The bands of C-H and N-H are located at 1024 $\mathrm{cm}^{-1}$ and $1155 \mathrm{~cm}^{-1}$,respectively, whereas the band of the $\mathrm{C}-\mathrm{H}$ out of plane deformation vibration is observed at $961 \mathrm{~cm}^{-1}$ [10]. Despite the variation in the dopant concentration, all five pyrrole polymers exhibited nearly the same FTIR absorption characteristics. The shoulder peak of PPy at 1003 $\mathrm{cm}^{-1}$ became more intense for the sample doped with $0.5 \mathrm{M}$ TSA. This finding suggests that the sulfonic acid groups were introduced into the polymer backbone [15].

\subsection{X-Ray Diffraction Analysis}

One of the most important techniques used for particle characterization is XRD. This method is typically used to analyze the morphology, size and shape of particles. Random patterns of particles will give rise to certain $\mathrm{X}$-ray diffraction [16]. The typical X-ray diffraction patterns for PPy particles synthesized with different concentrations of TSA are shown in Fig. 2. All of the XRD spectra of the PPy samples and the corresponding composites show broad peaks over the range $2 \theta=15-30$ o and an amorphous structure. The results are similar to those reported in previous studies [9, 17]. The PPy sample synthesized in the absence of TSA demonstrated a peak centered at $2 \theta=24.9^{\circ}$. The PPy/TSA composites (b), (c), (d), and (e) exhibited several broad peaks that are similar to those of PPy along, but with different peaks possitions. The PPy samples synthesized with $0.01 \mathrm{M}, 0.05 \mathrm{M}$, and $0.1 \mathrm{M}$ TSA showed peaks centered at $2 \theta=26.6^{\circ}$, whereas the sample synthesized with $0.5 \mathrm{M}$ TSA showed a peak centered at $2 \theta=26.0^{\circ}$.

In addition to the peak shift from $24.9^{\circ}$ to $26.6^{\circ}$ Fig. 2 (b-e) also demonstrates that the peak that formed was sharper than that shown in Fig. 2a. Furthermore, Fig. $2 \mathrm{c}$-d shows the peak area at $19.5^{\circ}$ This finding indicates that the TSA dopant was present in the structure of PPy. The presence of a dopant can generally cause changes in the position and shape of X-ray diffraction peaks. Such changes are caused by corresponding changes in the shape, size and density of the particles examined [18].

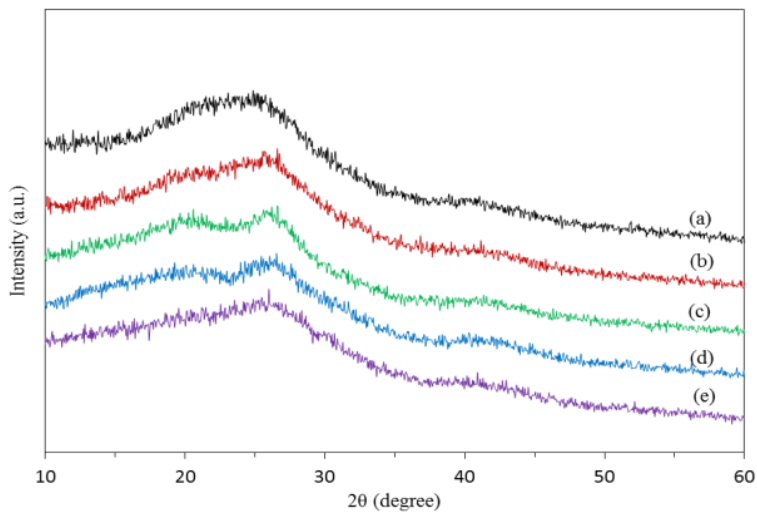

Fig. 2. X-ray diffraction patterns of PPy undoped (a); PPy synthesized at $0.01 M(b), 0.05 M(c), 0.1 M(d)$, and $0.5 \mathrm{M}(\mathrm{e}) \mathrm{TSA}$ concentrations.

\subsection{UV-Visible Analysis}

The UV-vis spectra of the samples were recorded in water and are presented in Fig.3. The absorption spectra of the samples showed two distinct bands at approximately $270 \mathrm{~nm}$ and $470 \mathrm{~nm}$ 
corresponding to the $\pi-\pi^{*}$ transition band. The absorption band at $470 \mathrm{~nm}$ was caused by the transition from the valence band to the most dominant bipolaron band [19]. The peak formed at approximately $470 \mathrm{~nm}$ is in accord with findings reported by Rai et al. (2008) [14] Liao et al. (2012) [13].

A shift in the peak at approximately $270 \mathrm{~nm}$ is observed between Fig. 3a-b and 3c; whereas Fig. 3ab shows a broad peak, Fig. 3c shows a sharp peak. This shift is related to the presence of TSA dopant in the polymer structure of the polypyrrole nanoparticles. The region around $270 \mathrm{~nm}$ in the UVvis spectra indicates a $\pi-\pi^{*}$ transition of the benzoid ring due to the TSA. The results shown agreed with those reported by Basavaiah et al. (2013) [15]. Fig. 3a shows that the dopant TSA had not yet been added, whereas Fig. $3 \mathrm{~b}$ shows that the TSA had begun to be added, although at a low concentration of $0.01 \mathrm{M}$. When the dopant concentration increased to $0.05 \mathrm{M}$, peak shifts and the formation of a sharp peak were observed, as indicated in Fig. 3c. The results are in accord with the statement made by Navale et al. (2014) indicating that the interaction between polypyrrole particles and dopant molecules may cause peak shifts in UV-vis spectra [20].

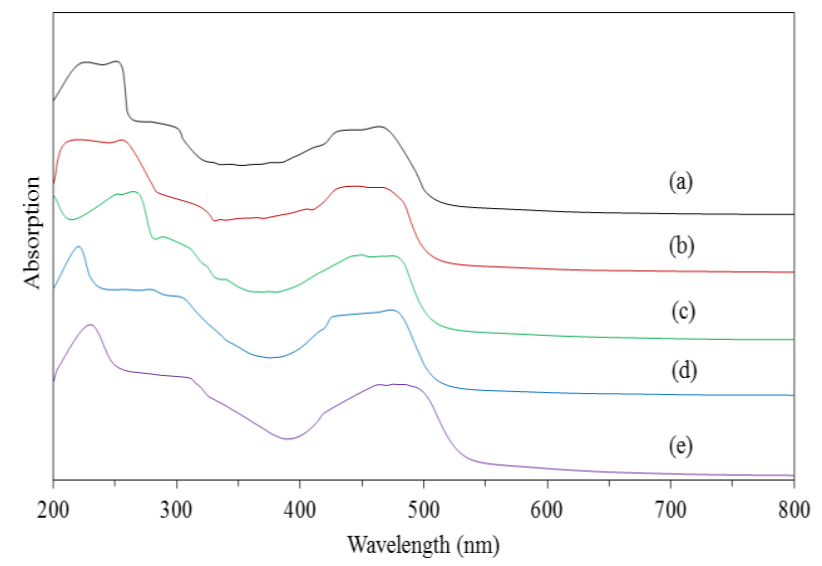

Fig. 3. UV-vis absorption of PPy undoped (a); PPy synthesized at $0.01 \mathrm{M}(\mathrm{b}), 0.05 \mathrm{M}(\mathrm{c}), 0.1 \mathrm{M}(\mathrm{d})$, and 0.5 M(e) TSA concentrations.

However, when the dopant concentrations were increased beyond $0.05 \mathrm{M}$, the peak shifted to approximately $250 \mathrm{~nm}$. This shift indicates changes in the structure of the polypyrrole nanoparticles. Increasing the TSA dopant concentration causes a change in the acidity of the solution because TSA is a strong acid [21]. The acidification of the solution thus damages the coordination bond formed between PPy and TSA. In this study, this condition led to changes in the structure of the PPy nanoparticles.
This results is in accord with that reported by Yang et al. (2012), who indicated that changes made to the solvent used to disperse polymer particles can effect the conformation of polymer chains and the lengths of conjugated segments, thereby leading to changes in the energy band gaps [22].

\subsection{SEM}

SEM images of the PPy particles doped with different concentration of TSA are presented in Fig. 4. It can be observed that each concentration of TSA gave rise to granular PPy particles, although the particle size varied with the dopant concentration. The PPy/TSA (images b-e) particles are clearly smaller than the undoped PPy particles (images a). The average particle size varied with the surfactant/dopant ratio because this ratio affects the viscosity of the solution and because the surfactant and dopant likely adsorb onto growing polypyrrole particles [23].

The particle size was also confirmed by a particle size analyzer. Table 1 shows the EDX result obtained for the samples. Higher sulfur content was detected for sample (c), in which the PPy particles were doped with $0.05 \mathrm{M}$ TSA. As expected, the samples containing high contents of sulfonic groups showed higher conductivities (Table 1).

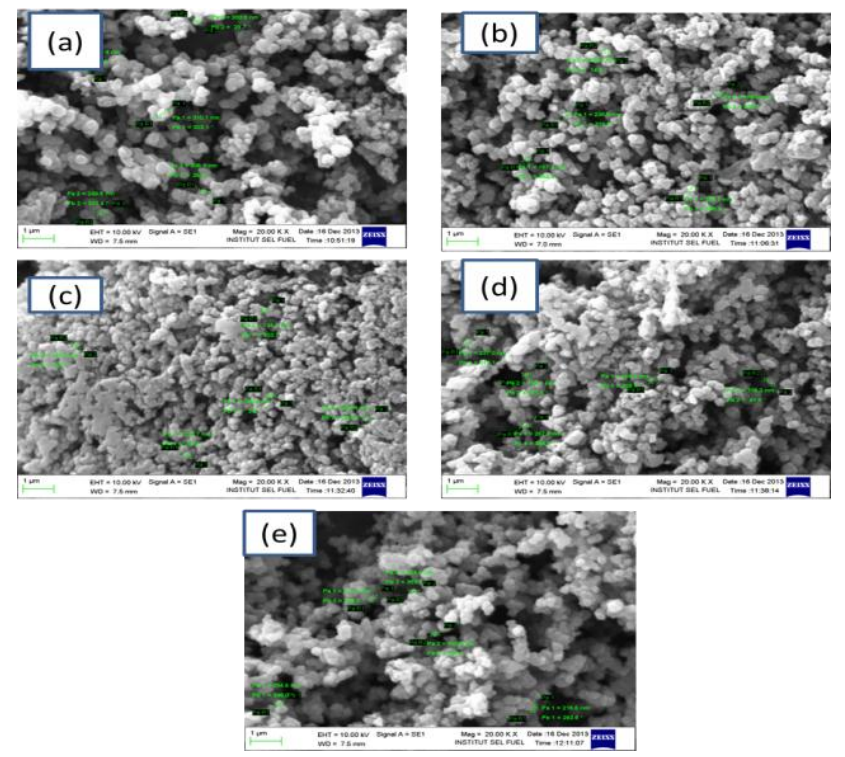

Fig. 4. SEM graph of PPy undoped (a); PPy synthesized at $0.01 M(b), 0.05 M(c), 0.1 M(d)$, and $0.5 M(e) T S A$ concentrations.

The data shown in Table 1 demonstrate that low a dopant concentration, i.e., $0.01 \mathrm{M}$, yielded a low sulfur content, which increased as the dopant concentration was elevated to $0.05 \mathrm{M}$. However, beyond $0.05 \mathrm{M}$, the sulfur content began to decrease. 
This trend suggests that TSA was not actually bound to PPy particles but only formed weak coordination bonds, as shown in Fig. 5.

Table 1. EDX result of the samples at various concentration of TSA dopant

\begin{tabular}{|l|l|l|l|l|l|l|l|l|l|l|}
\hline \multirow{2}{*}{ Element } & \multicolumn{2}{|c|}{ (a) } & \multicolumn{2}{c|}{ (b) } & \multicolumn{2}{c|}{ (c) } & \multicolumn{2}{c|}{ (d) } & \multicolumn{2}{c|}{ (e) } \\
\cline { 2 - 12 } & $\mathrm{Wt} \%$ & $\mathrm{At} \%$ & $\mathrm{Wt} \%$ & $\mathrm{At} \%$ & $\mathrm{Wt} \%$ & $\mathrm{At} \%$ & $\mathrm{Wt} \%$ & $\mathrm{At} \%$ & $\mathrm{Wt} \%$ & $\mathrm{At} \%$ \\
\hline CK & 54.39 & 67.36 & 47.74 & 63.01 & 51.52 & 68.41 & 54.72 & 69.06 & 56.19 & 73.68 \\
\hline NK & 16.15 & 17.16 & 14.62 & 16.55 & 13.45 & 15.31 & 12.58 & 13.61 & 11.13 & 12.51 \\
\hline OK & 13.41 & 13.41 & 15.37 & 15.23 & 9.68 & 9.65 & 12.99 & 12.31 & 9.59 & 9.44 \\
\hline SK & 4.64 & 2.15 & 8.25 & 4.08 & 10.99 & 5.46 & 8.86 & 4.19 & 6.13 & 3.01 \\
\hline AuM & 11.41 & 0.86 & 14.02 & 1.13 & 14.36 & 1.16 & 10.85 & 0.84 & 16.96 & 1.36 \\
\hline
\end{tabular}

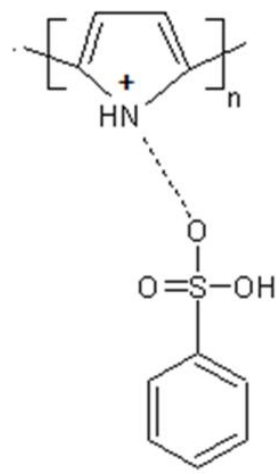

Fig. 5. Molecule Structure of PPy/TSA nanoparticles

At concentrations above $0.05 \mathrm{M}$, because the dopant concentration is already saturated, the equilibrium is shifted. The same condition also occurs in the synthesis of polypyrrole with anionic spherical polyelectrolyte brushes as the dopant (PPy/ASPB), as reported by Su et al. (2012). In these particles, the oxygen atom of the dopant will coordinate with the functional groups of $\mathrm{NH}$ on the pyrrole structure [24].

\subsection{Particle Size}

During the preparation of nanoparticles, the surfactant concentration plays an important role because it affects the size, distribution and morphology of the resulting particles [25]. Microemulsion is one technique used to form polymer nanoparticles. In this technique, particles are allowed to aggregate and form a surfactant system. There are two reasons why a surfactant is used for polymerization. First, a surfactant allows for the creation of a vessel by forming a microemulsion of micelles, in which monomers are localized in small capsules formed by surfactant molecules. Second, surfactants improve the physical properties of polymers, such as their conductivity, stability and solubility in organic solvents $[14,22,26]$

In this investigation, it was observed that TSA plays a significant role as a dopant and surfactant in the formation of PPy/TSA nanostructures. This finding is similar to that reported by Basavaiah (2011) for the synthesis of tetraaniline nanoparticles. The bearing of methyl groups and hydrophilic $\mathrm{SO}_{3} \mathrm{H}$ groups by TSA allows for the formation of micelles in water. These micelles serve as soft templates for the growth of PPy nanoparticles, where the presence of hydrophilic methyl groups from TSA helps discourage aggregation due to the repulsive forces exerted by the nanoparticles [15].

Fig. 6 shows the size distribution of TSA-doped polypyrrole particles formed at different TSA concentrations. The various sizes of the particles were determined to be $712-1484 \mathrm{~nm}, 190-531 \mathrm{~nm}$, 24-51 nm, $164-255 \mathrm{~nm}$, and 190-712 nm for TSA concentrations of $0.0 \mathrm{M}, 0.01 \mathrm{M}, 0.05 \mathrm{M}, 0.1 \mathrm{M}$, and $0.5 \mathrm{M}$ respectively. The size of the polypyrrole particles decreased with the increase in the TSA concentration. The smallest particle size was observed for $0.05 \mathrm{M}$ TSA.

As clearly demonstrated in Fig. 7, the dopant concentration affected the size of the particles, in agreement with a study by Devi et al. (2013) [27]. The average size of the PPy particles formed in the absence of TSA was very large, i.e., $\pm 1 \mu \mathrm{m}$, compared with that of the particles formed in the presence of TSA. Nevertheless, after the presence of the dopant was first detected, a concentration of 0.05 $\mathrm{M}$, the average particle size decreased to $32.7 \mathrm{~nm}$.

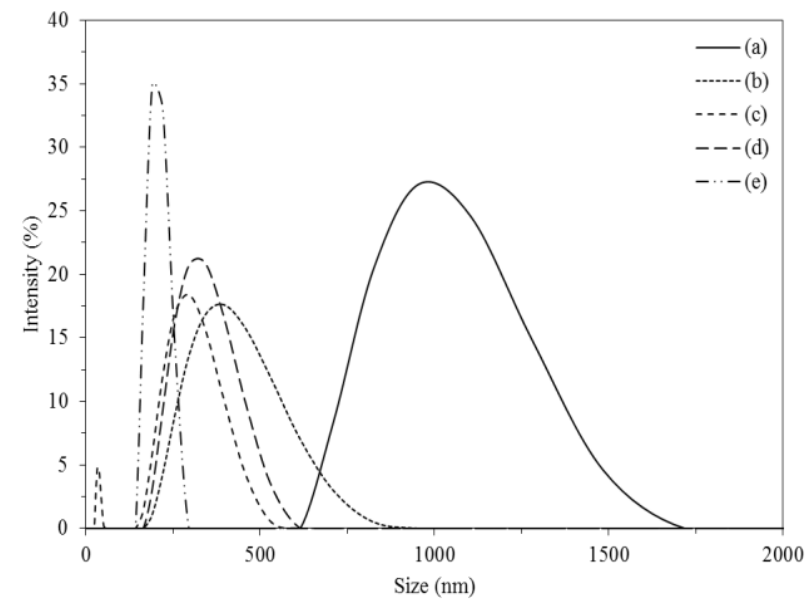

Fig. 6. Particles size analysis of PPy undoped (a); PPy synthesized at $0.01 \mathrm{M}(b), 0.05 \mathrm{M}(\mathrm{c}), 0.1 \mathrm{M}(\mathrm{d})$, and 0.5 $M(e)$ TSA concentrations. 


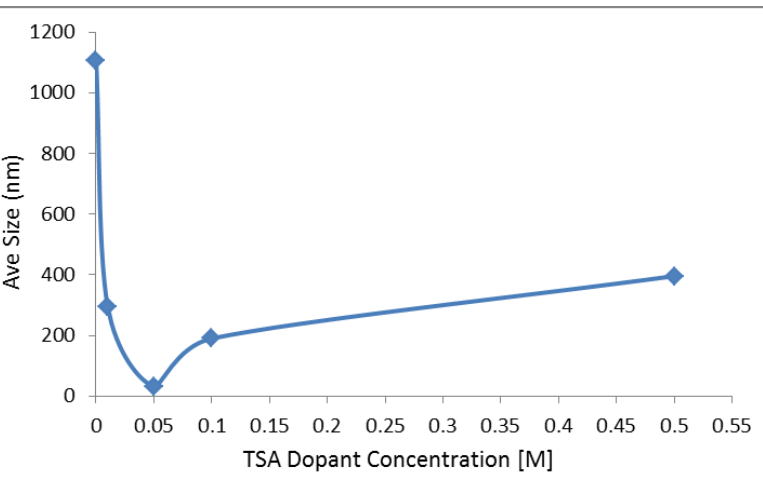

Fig. 7. Average size of PPy/TSA particles at different concentration of TSA dopant

This phenomenon led to the formation of microemulsions due to the interaction between water and TSA, which also acted as a surfactant. Consequently, PPy monomers were localized in the microemulsions, and further polymerization occurred upon the addition of APS, which served as an oxidant [21, 22, 26-28].

In the absence of surfactant molecules, PPy particles easily aggregated due to dipole-dipole van der Waals forces between the particles, thus producing a large particles and a non-uniform size distribution [15, 29]. Fig. 8 shows the proposed formation mechanism of polypyyrole/TSA nanoparticles.

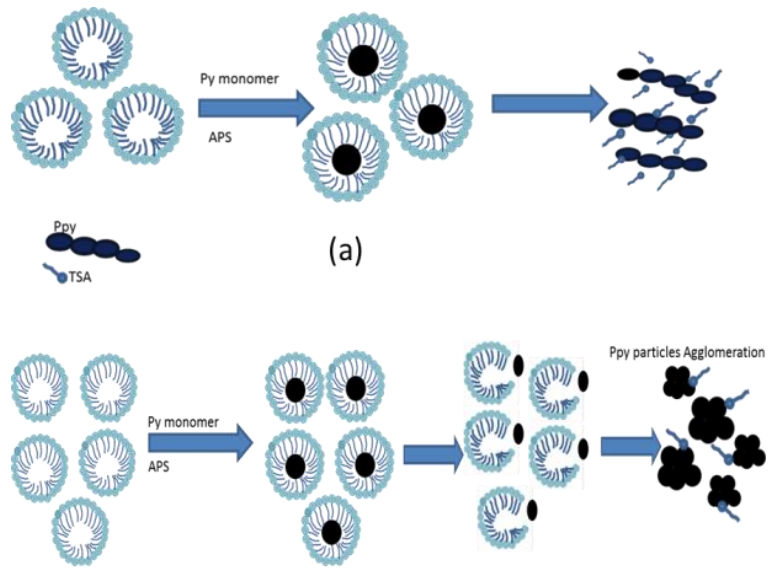

(b)

Fig. 8. Formation mechanism of PPy/TSA nanoparticles proposed, (a) at low concentration of TSA and (b) on high concentration of TSA

Based on the graph shown in Fig. 7, the dopant concentration was detectable in excess of $0.05 \mathrm{M}$; however, in this experiment between $0.1 \mathrm{M}$ and 0.5 $M$ were used, thus resulting in even larger particle sizes. This contradiction is due to the formation of smaller polymer particles, which are easier to dissolve at regular intervals. This process causes the microemulsions formed to become unstable and triggers the breakdown of existing microemulsions. Such a mechanism was also reported by Tigges et al. (2010) [30]. As a result, the particles will interact with each other regardless of dipole-dipole interactions and van der Waals forces to form larger particles measuring, on average, $190 \mathrm{~nm}$ and 396.1 $\mathrm{nm}[15,29]$. This same behavior can be observed in Fig. 4.

\subsection{Thermo gravimetric analysis}

Thermogravimetric analysis (TGA) is used to study the thermal stability of polymers and monitor the weight loss of materials as a function of temperature [31]. Fig. 9 shows the TGA curve of the PPy/TSA samples. Weight losses of approximately $9 \%$ to $12 \%$ are associated with the PPy and PPy/TSA particles at approximately $100^{\circ} \mathrm{C}$ and can be attributed to the hygroscopic character of the samples; indeed, at approximately $100^{\circ} \mathrm{C}$, the remaining water held in the PPy/TSA nanoparticles is evaporated [22]. As the temperature was raised, to approximately $200^{\circ} \mathrm{C}$, the PPy/TSA nanoparticles began to break down. Similar findings have been reported in previous studies [13].

A comparison of Fig.s 9a, 9b and 9e reveals that the PPy particles exhibited better thermal stability in the presence of dopant molecules than in their absence. This result suggests that the presence of dopant molecules could enhance the thermal stability of the nanoparticles. It is know that sulfonic groups exhibit good thermal stability if they form complexes with counter ions.

However, as the concentration of the dopant was raised, the thermal stability approached that of the PPy nanoparticles with no dopant, which indicates that the dopant did not actually reside in the PPy structures. These data also support the results obtained from EDX, UV-Vis, FTIR and XRD, as previously indicated. Similar findings were reported by Wang et al. (2011) [7]. Above $450^{\circ} \mathrm{C}$, the main backbone of PPy decomposed and the rings of PPy were opened [24]. 


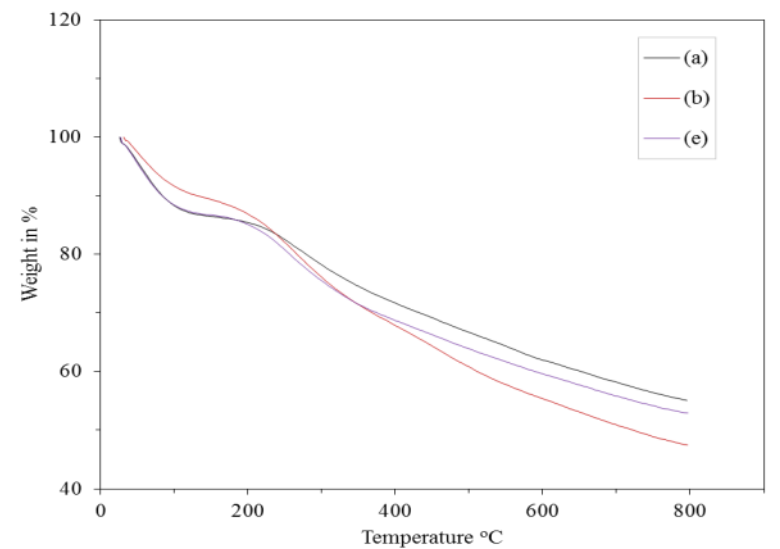

Fig. 9. Thermogravimetric analysis for PPy synthesized at (a) $0.01 \mathrm{M}$, (b) $0.05 \mathrm{M}$ and (e) $0.5 \mathrm{M}$ TSA dopant concentration

\subsection{Electrical conductivity}

The character and concentration of the dopant used can affect the rate of particle formation, the particle size, the atomic distribution, the homogeneity and the structure of particles that are synthesized. Furthermore, morphology is very important in determining the conductivity and mechanical properties of particles [32].

The electrical conductivity of the PPy/TSA samples is presented in Table 2. As the dopant concentration was increased from $0.01 \mathrm{M}$ to $0.05 \mathrm{M}$, the conductivity increased from $6.1 \times 10^{-2} \mathrm{~S} / \mathrm{cm}$ to 1.3 $\mathrm{x} 10^{-1}$. As the dopant concentration was increased to 0.1 and 0.5 the conductivity decreased. At a dopant concentration $0.05 \mathrm{M}$, the size of the polypyrrole/TSA particles was very small, homogeneous and narrower in distribution, as indicated by the SEM images, than at other dopant concentrations. This small particle size indicates that the surface area of the polypyrrole particles was high, thereby affecting the electron density. A high electron density indicates that electron motion and thus DC conductivity $(\sigma)$ are correspondingly high [33].

Table 2. Conductivity values of polypyrrole at different concentration of TSA at room temperature

\begin{tabular}{ll}
\hline Concentration of TSA & Conductivity $\left(\mathrm{S} \mathrm{cm}^{-1}\right)$ \\
\hline $\mathrm{M}$ & $3.4 \times 10^{-3}$ \\
$\mathrm{M}$ & $6.1 \times 10^{-2}$ \\
$0.05 \mathrm{M}$ & $1.3 \times 10^{-1}$ \\
$0.1 \mathrm{M}$ & $9.6 \times 10^{-2}$ \\
$0.5 \mathrm{M}$ & $2.6 \times 10^{-2}$ \\
\hline
\end{tabular}

The correlation between particle size and DC conductivity is shown in Table 2. As the particle size was reduced, the overall surface area was increased, thus leading to a high coulomb efficiency and, in turn, an increase in the electron transfer and conductivity of the particles [34].

Electron motion in the particles occurs through a jumping or hopping mechanism. Electrons travel through vacancies in the particles' crystal structure. In small particles that are compact and homogeneous, electrons travel more easily than in large, non-uniform particles. Along coarse, nonuniform particles, there arelarge gaps between the edges of large particles, as shown in Fig. 4a, b, d and e. Such gaps cause the energy required for electrons to jump to increase and thus inhibits the movement of electrons [35]. The hopping mechanism is illustrated in Fig. 10.

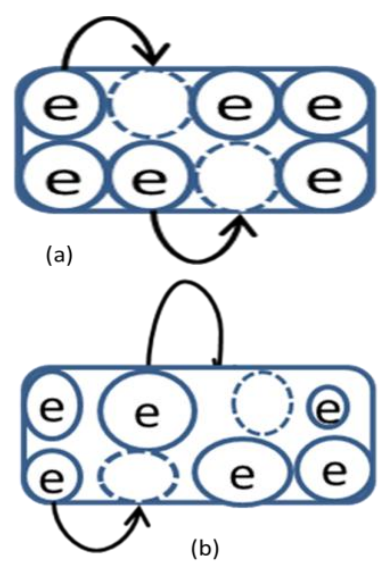

Fig. 10. Illustration of electron movement of smaller particles, compact and homogenous (a), rough and not uniform (b).

\section{Conclusions}

PPy/TSA particles were synthesized at various concentrations of TSA. FTIR imaging confirmed that the dopant used was present in the structure of the conductive polypyrrole particles. However, the dopant TSA did not actually penetrate into the particles but was only coordinated on their surface, as indicated by EDX. In this study, it was found that the dopant concentration affected the resulting particles' size and electrical conductivity, as supported by SEM imaging. XRD spectra demonstrate that amorphous composite polypyrrole particles were formed and that the presence of the TSA dopant did not alter the morphology of the polypyrrole particles. Furthermore, the PPy/TSA nanoparticles showed good thermal stability, withstanding temperatures of up to $250^{\circ} \mathrm{C}$ as indicated by thermogravimetric analysis. Based on the results obtained in this study, PPy/TSA nanoparticles show promising application as components of fuel cells. 


\section{Acknowledgments}

The researchers give many thanks in appreciation to the National University of Malaysia and the Government of Malaysia for the financial support they provided through research grants GGPM-2013-044, FRGS/2/2013/TKO4/UKM/02/3 and DIP-2014-002. Researchers also say thank you to Mercu Buana University who has provided support for the research in progress.

\section{References}

[1] K. Qi, Y. Qiu, Z. Chen, X. Guo, Corrosion of conductive polypyrrole: Effects of environmental factors, electrochemical stimulation, and doping anions, Corrosion Science, 60 (2012) 50-58.

[2] L.-X. Wang, X.-G. Li, Y.-L. Yang, Preparation, properties and applications of polypyrroles, Reactive and Functional Polymers, 47 (2001) 125-139.

[3] H. Zhao, L. Li, J. Yang, Y. Zhang, Nanostructured polypyrrole/carbon composite as Pt catalyst support for fuel cell applications, Journal of Power Sources, 184 (2008) 375-380.

[4] S.-Y. Huang, P. Ganesan, B.N. Popov, Development of conducting polypyrrole as corrosion-resistant catalyst support for polymer electrolyte membrane fuel cell (PEMFC) application, Applied Catalysis B: Environmental, 93 (2009) 75-81.

[5] C. Feng, L. Ma, F. Li, H. Mai, X. Lang, S. Fan, A polypyrrole/anthraquinone-2, 6-disulphonic disodium salt (PPy/AQDS)-modified anode to improve performance of microbial fuel cells, Biosensors and Bioelectronics, 25 (2010) 15161520.

[6] H. Park, Y. Kim, Y.S. Choi, W.H. Hong, D. Jung, Surface chemistry and physical properties of Nafion/polypyrrole/Pt composite membrane prepared by chemical in situ polymerization for DMFC, Journal of Power Sources, 178 (2008) 610-619.

[7] Y. Wang, C. Yang, P. Liu, Acid blue AS doped polypyrrole (PPy/AS) nanomaterials with different morphologies as electrode materials for supercapacitors, Chemical Engineering Journal, 172 (2011) 1137-1144.

[8] J. Upadhyay, A. Kumar, Structural, thermal and dielectric studies of polypyrrole nanotubes synthesized by reactive self degrade template method, Materials Science and Engineering: B, 178 (2013) 982-989.

[9] J. Hazarika, A. Kumar, Controllable synthesis and characterization of polypyrrole nanoparticles in sodium dodecylsulphate (SDS) micellar solutions, Synthetic Metals, 175 (2013) 155-162.

[10] Y. Cao, P. Smith, A.J. Heeger, Counter-ion induced processibility of conducting polyaniline, Synthetic Metals, 57 (1993) 35143519.

[11] H.T. Ham, Y.S. Choi, N. Jeong, I.J. Chung, Singlewall carbon nanotubes covered with polypyrrole nanoparticles by the miniemulsion polymerization, Polymer, 46 (2005) 6308-6315.

[12] Y. Liu, Y. Chu, L. Yang, Adjusting the innerstructure of polypyrrole nanoparticles through microemulsion polymerization, Materials chemistry and physics, 98 (2006) 304-308.

[13] Y. Liao, X. Wang, W. Qian, Y. Li, X. Li, D.-G. $\mathrm{Yu}$, Bulk synthesis, optimization, and characterization of highly dispersible polypyrrole nanoparticles toward protein separation using nanocomposite membranes, Journal of colloid and interface science, 386 (2012) 148-157.

[14] A. Reung-U-Rai, A. Prom-Jun, W. Prissanaroon-Ouajai, S. Ouajai, Synthesis of highly conductive polypyrrole nanoparticles via microemulsion polymerization, Journal of Metals, Materials and Minerals, 18 (2008) 2731.

[15] K. Basavaiah, Y. Pavankumar, A.V.P. Rao, A facile one-step synthesis of PTSA-doped tetraaniline nanostructure/magnetite nanoparticles via self-assembly method, Journal of Nanostructure in Chemistry, 3 (2013) 74.

[16] B. Akbari, M.P. Tavandashti, M. Zandrahimi, Particle Size Characterization of NanoparticlesA Practicalapproach, Iranian Journal of Materials Science and Engineering, 8 (2011) 48-56.

[17] C. MA, S. Shashwati, Synthesis and characterization of polypyrrole (PPy) thin films, Soft Nanoscience Letters, 2011 (2011).

[18] H.K. Chitte, N.V. Bhat, A.V. Gore, G.N. Shind, Synthesis of Polypyrrole Using Ammonium Peroxy Disulfate (APS) as Oxidant Together with Some Dopants for Use in Gas Sensors, Materials Sciences \& Applications, 2 (2011).

[19] H.-Y. Woo, W.-G. Jung, D.-W. Ihm, J.-Y. Kim, Synthesis and dispersion of polypyrrole nanoparticles in polyvinylpyrrolidone emulsion, Synthetic Metals, 160 (2010) 588-591.

[20] S. Navale, A. Mane, A. Ghanwat, A. Mulik, V. Patil, Camphor sulfonic acid (CSA) doped polypyrrole (PPy) films: Measurement of microstructural and optoelectronic properties, Measurement, 50 (2014) 363-369. 
[21] M.M. Demir, R. Munoz-Espi, I. Lieberwirth, G. Wegner, Precipitation of monodisperse $\mathrm{ZnO}$ nanocrystals via acid-catalyzed esterification of zinc acetate, Journal of Materials Chemistry, 16 (2006) 2940-2947.

[22] C. Yang, X. Wang, Y. Wang, P. Liu, Polypyrrole nanoparticles with high dispersion stability via chemical oxidative polymerization in presence of an anionic-non-ionic bifunctional polymeric surfactant, Powder Technology, 217 (2012) 134-139.

[23] P. Jayamurgan, V. Ponnuswamy, S. Ashokan, T. Mahalingam, The effect of dopant on structural, thermal and morphological properties of DBSA-doped polypyrrole, Iranian Polymer Journal, 22 (2013) 219-225.

[24] N. Su, H. Li, S. Yuan, S. Yi, E. Yin, Synthesis and characterization of polypyrrole doped with anionic spherical polyelectrolyte brushes, Express Polymer Letters, 6 (2012) 697.

[25] C. Tojo, M.d. Dios, F. Barroso, Surfactant effects on microemulsion-based nanoparticle synthesis, Materials, 4 (2010) 55-72.

[26] J. Stejskal, M. Omastová, S. Fedorova, J. Prokeš, M. Trchová, Polyaniline and polypyrrole prepared in the presence of surfactants: a comparative conductivity study, Polymer, 44 (2003) 1353-1358.

[27] P. Devi, M. Singla, Effect of surfactant concentration, solvents and particle size on $\prod-$ A isotherm of silica nanoparticles, Materials Letters, 107 (2013) 107-110.

[28] H. Zheng, Y. Jiang, J. Xu, Y. Yang, The characteristic properties of PEDOT nanoparticle based on reversed micelle method, Science China Technological Sciences, 53 (2010) 2355-2362.

[29] N.I. Lebovka, Aggregation of charged colloidal particles, Polyelectrolyte Complexes in the Dispersed and Solid State I, Springer2014, pp. 57-96.

[30] B. Tigges, T. Dederichs, M. Möller, T. Liu, W. Richtering, O. Weichold, Interfacial properties of emulsions stabilized with surfactant and nonsurfactant coated boehmite nanoparticles, Langmuir, 26 (2010) 17913-17918.

[31] M. Jakic, N.S. Vrandecic, I. Klaric, Thermal degradation of poly (vinyl chloride)/poly (ethylene oxide) blends: Thermogravimetric analysis, Polymer Degradation and Stability, 98 (2013) 1738-1743.

[32] H. Eisazadeh, Studying the characteristics of polypyrrole and its composites, World Journal of Chemistry, 2 (2007) 67-74.
[33] F.A. Saad, M.M. Abou-Sekkina, A.M. Khedr, F.G. El-Metwaly, Synthesis, Stability and DCelectrical Conductivity of Vanadium and Chromium Dual Doped LiMn 2 O 4 Spinals as Cathode Material for Use in Lithium Rechargeable Batteries, International Journal of Electrochemical Science, 9 (2014).

[34] C.-H. Lu, S.-W. Lin, Influence of the particle size on the electrochemical properties of lithium manganese oxide, Journal of power sources, 97 (2001) 458-460.

[35] S.A. Makhlouf, M.A. Kassem, M. AbdelRahim, Particle size-dependent electrical properties of nanocrystalline $\mathrm{NiO}$, Journal of materials science, 44 (2009) 3438-3444. 